\title{
e-Phaïstos
}

e-Phaïstos Revue d'histoire des techniques / Journal of the history of technology

IV-2 | 2015

Patrimoines de l'eau

\section{Les usines élévatoires du Sénégal entre 1898 et}

1920

Senegalese Pumping Stations from 1898 to 1920

\section{Salif Diedhiou}

\section{(2) OpenEdition}

Journals

Édition électronique

URL : http://journals.openedition.org/ephaistos/757

DOI : 10.4000/ephaistos.757

ISSN : 2552-0741

Éditeur

IHMC - Institut d'histoire moderne et contemporaine (UMR 8066)

Édition imprimée

Date de publication : 1 octobre 2015

Pagination : 54-59

ISSN : 2262-7340

Référence électronique

Salif Diedhiou, «Les usines élévatoires du Sénégal entre 1898 et 1920 », e-Phaïstos [En ligne],

IV-2 | 2015, mis en ligne le 28 novembre 2016, consulté le 20 avril 2019. URL : http://

journals.openedition.org/ephaistos/757 ; DOI : 10.4000/ephaistos.757

Tous droits réservés 


\title{
Les usines élévatoires du Sénégal entre 1898 et 1920
}

\author{
Salif Diedhiou \\ Master Erasmus Mundus TPTI, \\ promotion 3 《Conserverasmus » \\ Doctorant, EPHE, PresHesam
}

\section{Introduction}

L'outillage économique ${ }^{1}$ mis en place dans les colonies françaises pour servir de levier à leur développement, constitue un ensemble d'infrastructures. Il consiste à créer les conditions favorables à l'exploitation des ressources des colonies au profit de la métropole.

Pour ce faire, l'État colonisateur met en place un système d'administration ${ }^{2}$ et de gestion des territoires occupés. Ceci conduit à la création du service des Travaux publics au Sénégal par les arrêtés du 3 juillet 1841 et du 22 septembre 1849 . Avant, il y avait le service du génie avec des attributs similaires.

En 1898 les missions du service des Travaux Publics ont été clairement définies, empruntant certaines dispositions de la législation métropolitaine3. Ces missions consistent à :

- $\quad$ préparer des plans, projets, devis et cahier des charges des travaux publics autres que ceux qui entrent dans le cadre des attributions militaires, de la conduite et de la surveillance de ces travaux, de la constatation des dépenses qui en résultent et de la préparation des pièces nécessaires à leur paiement ; - entretenir et conserver les travaux publics, notamment les routes, chemins, rues et places faisant partie de la voirie, les ouvrages d'art et les plantations qui en dépendent, les quais, cales de déchargement, jetées établies aux frais de la colonie, les bâtiments civils, les machines, réservoirs et conduites de distribution d'eau établies dans les mêmes conditions, les phares et balises et aménagement des marées ;

- $\quad$ préparer des arrêtés d'alignement le long des routes et chemins faisant partie de la grande voirie ;

- conserver des terrains faisant partie des domaines de la colonie, des rapports sur la délimitation, l'estimation, la concession ou la vente de ces terrains, et de la rédaction ou du dépôt des titres et plans qui y sont relatifs ;

- contrôler des travaux exécutés par les concessionnaires pour le compte de la colonie ;

- contrôler des travaux exécutés par les municipalités avec le concours des fonds de la colonie.

Le service est dirigé par un ingénieur civil qui assume les fonctions de directeur des travaux publics du Sénégal et à ce titre est membre du conseil privé de la colonie. Ainsi, le développement des transports ferroviaires et routiers, l'assainissement et l'adduction de l'eau potable des villes, l'électrification, l'urbanisme et l'habitat ont été conçus et pilotés par ce service animé par des ingénieurs sortis des écoles de la métropole et affectés dans les colonies. Saint-Louis et Dakar sont deux villes modèles de l'encadrement très rigoureux dans l'administration des secteurs de l'eau et de l'électricité. L'eau, élément vital pour les nombreux expatriés français résidant dans la colonie et confrontés aux maladies mortelles liées à sa consommation, a fait l'objet d'une attention particulière dans l'application de l'ingénierie déployée au plan local. 


\section{L'adduction d'eau pré-électrique : cas de St-Louis, Rufisque et Dakar}

La position côtière des principaux centres de la colonie du Sénégal, notamment Saint-Louis, Rufisque et Dakar, rend plus que préoccupante la distribution de l'eau potable à une population de plus en plus importante. Devant cette exigence, l'administration décide de prendre à bras le corps la recherche de solutions. Elle commandite des études dès 1898. Cette mission a été confiée au service des Travaux Publics qui présente les projets définitifs au Conseil Général dans sa session de mai 1899. Après leur adoption par cette assemblée, la mise en exécution de ces projets a été décidée à la suite de leur approbation par le département des colonies. Saint-Louis, de part son statut de première capitale de la colonie abritant une communauté importante d'européens, a été le premier champ d'expérimentation.

\section{St-Louis et sa dépendance au fleuve Sénégal}

La ville de Saint-Louis a la particularité d'être côtière de l'océan Atlantique (eau salée) et à l'embouchure du fleuve Sénégal dont la source se trouve au Fouta Djalon en Guinée (eau douce). Son alimentation en eau est conditionnée par les deux saisons qui existent en Afrique : la saison des pluies (fin juillet-fin novembre) correspond à l'eau douce du fleuve et la saison sèche (décembre-juin) est celle de l'eau salée. En conséquence, il existe deux systèmes distincts de service d'alimentation d'eau de la ville basés sur deux marigots (petits bras du fleuve Sénégal) : Kor $(2,5 \mathrm{~km})$ et Kassak $(18 \mathrm{~km})$. Ceci amène les ingénieurs du génie civil à concevoir deux systèmes d'alimentation de la ville en eau potable. Chaque système est doté d'un appareillage spécifique.

\section{$\underline{\text { Service d'alimentation en saison pluvieuse }}$}

Ce service se caractérise par la prise d'eau au marigot de Kor, alimenté par les eaux du fleuve en période douce, avec un débit de $4000 \mathrm{~m}^{3} /$ seconde. Une usine élévatoire est implantée à Korr pour refouler les eaux dans les réservoirs de Sor d'où elles se rendent dans le réseau de la ville par gravité.

L'usine élévatoire de Korr est très modeste. Elle est composée d'une machine verticale de dix chevaux. Celle-ci est alimentée par une chaudière Field qui actionne une pompe à double effet. L'usine fonctionne nuit et jour pendant la période d'eau douce du fleuve afin d'assurer le besoin minimum des populations.

\section{Service d'alimentation en saison sèche}

A partir de janvier, quand les eaux du fleuve prennent un taux de salinité impropre à la consommation, le relais de l'alimentation se fait au niveau du marigot de Kassak. Le système d'alimentation de la ville part de l'usine élévatoire construite à Makhana. Le marigot se remplit d'eau pendant les crues. On l'isole en fermant les pertuis d'un barrage en maçonnerie situé en aval, dès que les eaux du fleuve commencent à baisser et à prendre un degré de salinité.

L'usine élévatoire de Makhana prend à ce moment le relais de celle de Korr pour sept mois et demi, assurant ainsi l'alimentation de la ville. Cette usine, mieux outillée que celle de Korr, est dotée d'une installation double: deux machines commandant chacune leurs pompes et deux chaudières.

Ces machines marchent séparément avec une capacité de refouler $2.200 \mathrm{~m}^{3} /$ jour. Mais la conduite de refoulement qui traverse la saline de Makhana sur 3000m subit des avaries qui ramènent la pression de 2200 à $1800 \mathrm{~m}^{3} / \mathrm{jour}$, ce qui est loin de couvrir les besoins. Au mois de mai, il $\mathrm{n}$ y a presque plus d'eau dans le marigot, créant des arrêts de plusieurs heures dans le fonctionnement de l'usine.

Pour pallier à cette situation, une autre petite usine élévatoire est construite à N'diol où s'arrêtent 
des conduites initialement prévues pour puiser l'eau de Richard Toll. Cette petite usine élévatoire de N'Diol, qui a fonctionné en juin-juillet 1898, envoie l'eau du marigot dans les bassins en maçonnerie de Makhana d'où les pompes la reprennent pour la refouler dans le réseau de la ville de Saint-Louis. En décidant de porter de 2000 à $4000 \mathrm{~m}^{3} /$ jour la capacité de refoulement de l'eau dans l'alimentation de la ville de Saint-Louis, le conseil général décide d'établir à Makhana et à Korr de nouvelles usines élévatoires plus puissantes que les premières et d'étendre le réseau de distribution d'eau pour desservir tous les quartiers de la ville. L'usine de N'Diol a été abandonnée pour insuffisance de rendement.

\section{Les fournisseurs d'équipements du service de distribution de l'eau 4}

Il faut tout un équipement pour assurer la distribution de l'eau dans la ville de Saint-Louis : des bâtiments aux réservoirs, en passant par les canalisations. Tout un arsenal dont la satisfaction demande l'intervention de divers fournisseurs. Des marchés de gré à gré ont été adjugés à des entreprises françaises résidant en métropole. Ceuxci concernent la robinetterie, la tôlerie et la tuyauterie.

La robinetterie et accessoires divers pour conduites d'eau a fait l'objet d'un marché de gré à gré adjugé à MM. MATHELIN et GARNIER, ingénieurs civils, demeurant à Paris rue Boursault, $\mathrm{n}^{\circ} 26$, qui se sont engagés à construire dans leurs ateliers de Lille (Nord) et à livrer à Bordeaux, dans les magasins de la marine, les objets de robinetterie et accessoires divers énumérés dans les clauses prédéfinies. Ce marché a été souscrit le 3 octobre 1887.

Le marché pour la fourniture à Saint-Louis de la tôlerie nécessaire à la construction de deux réservoirs d'eau et de leur couverture a été adjugé à MM. J. de Schryver et Cie, ingénieurs constructeurs à Haumont (Nord). Ils doivent construire dans leurs ateliers les tôles, cornières, fers spéciaux, rivets et boulons nécessaires à la construction de deux réservoirs d'eau et leur couverture selon les clauses. Ce marché est souscrit le 3 octobre 1887.

Quant au marché des tuyaux et objets en fonte nécessaire à l'établissement d'une conduite d'eau à Saint-Louis, il a été adjugé à Jules Leblanc, constructeur, demeurant à Paris, 52 rue du RendezVous. Il doit construire dans ses ateliers de Marquise, les tuyaux, les pièces de raccord, les gargouilles, les consoles et les trappes pour l'installation de la canalisation.

Le constructeur J. Leblanc était déjà chargé du projet de modification de la prise d'eau de Sor et Sant-Louis depuis 1884 par l'administration de la colonie. Il a produit des plans de conduites et le système d'élévation des eaux. Il a également fourni les machines à vapeur de l'usine élévatoire de Makhana, qui sont encore dans cette usine abandonnée depuis 1969. Ce sont les plus vieilles de l'Afrique noire.

Pour construire les pièces, tous les fournisseurs sont invités à se référer pour une partie aux dimensions et formes indiquées sur l'album des types, joint au marché passé par la Ville de Paris, le 16 janvier 1886. Les autres pièces seront conformes aux dessins types remis aux fournisseurs par le service technique de la colonie.

\section{Rufisque tire sa source des dunes}

Le système de distribution de l'eau à Rufisque est bien différent de celui appliqué à Saint-Louis. Ici, la source d'eau provient des dunes de sable situées à un kilomètre environ de la ville. En 1898, les mille européens et les villages indigènes créent un besoin d'alimentation d'une population estimée à dix mille habitants.

Jusqu'ici quelques rares privilégiés possèdent chez eux des citernes qui captent les eaux de pluies pendant l'hivernage pour une stricte alimentation durant la saison sèche. Les moins nantis vont chercher l'eau dans des puits creusés dans les dunes 
à un kilomètre de la ville.

Le sondage effectué dans ces dunes révèle une capacité de stock nécessaire à l'alimentation de la ville en eau potable. Six puits filtrants distants de $120 \mathrm{~m}$, d'un diamètre intérieur de $2,50 \mathrm{~m}$, qui plongent d'environ $6 \mathrm{~m}$ dans la nappe aquifère sont construits le long du marigot de Sangalcam. Leur fonçage a été effectué sur rouet métallique par le procédé de havage. Ils sont de deux groupes, chacun desservi par une conduite de 200mm de diamètre. Ceci forme un siphon qui part du puits le plus éloigné par une rampe de $2 \mathrm{~m}$ vers un puisard de $3 \mathrm{~m}$ de diamètre plus profond que les puits d'un mètre, dans lequel aspirent les pompes élévatoires. Ce système est conçu pour fournir $600 \mathrm{~m}^{3} /$ jour nécessaires à l'alimentation de la ville.

Sangalcam est à $9500 \mathrm{~m}$ de Rufisque avec une nappe aquifère à la côte de $14 \mathrm{~m}$ au-dessus du niveau des basses mers. Rufisque est au bord de la mer à environ $4 \mathrm{~m}$ au-dessus des basses eaux. Le sol s'élève continuellement de Sangalcam à Rufisque jusqu'à $35 \mathrm{~m}$ sur une distance de $4600 \mathrm{~m}$, puis descend progressivement jusqu'à $4 \mathrm{~m}$ au-dessus de la mer. Des réservoirs d'approvisionnement sont installés au point culminant d'où l'usine élévatoire les refoule en ville par simple gravité. La conduite de refoulement et celle d'adduction sont de $250 \mathrm{~mm}$.

Les machines élévatoires sont dotées d'une puissance qui leur permet de refouler les $600 \mathrm{~m}^{3} / \mathrm{jour}$ en 8 heures avec une machinerie commandée en double. Une locomobile et une pompe centrifuge assurent le pompage des eaux depuis Sangalcam pour assurer l'alimentation de la ville de Rufisque constituée en réseau maillé.

\section{Dakar, un système de distribution complexe}

La situation maritime de la ville de Dakar rend son service de distribution d'eau plus complexe. Trouver une réserve naturelle d'eau douce pouvant alimenter la ville toute l'année était une gageure. Deux bassins d'une contenance totale de $3600 \mathrm{~m}^{3}$ sont construits près des quais du port pour emmagasiner les eaux pluviales. Ces bassins sont alimentés par des galeries filtrantes construites dans la partie nord de la ville où les eaux pluviales recueillies suivent une pente naturelle jusqu'aux citernes les mettant à l'abri de l'évaporation. Mais elles sont loin de couvrir les besoins de la population en saison sèche. Pour augmenter la capacité de fourniture d'eau, un bassin captant a été creusé près de Hann, fournissant $30 \mathrm{~m}^{3} /$ jour. Les dunes sablonneuses de Hann constituent un réservoir important alimenté par les eaux de pluie. Des galeries filtrantes en maçonnerie y ont été aménagées avec des radiers en béton pour capter les eaux nécessaires à l'alimentation de Dakar.

Finalement, une usine élévatoire a été prévue à Hann près des galeries filtrantes, en 1900, pour refouler les eaux captées dans les réservoirs d'une contenance de $2000 \mathrm{~m}^{3}$. Il s'agit d'une usine à vapeur devant comprendre deux machines à vapeur avec chacune sa chaudière et ses pompes élévatoires fonctionnant séparément avec une capacité de refoulement de $100 \mathrm{~m}^{3} / \mathrm{jour}$. La partie mécanique a été adjugée à MM. Piguet \& Cie de Lyon 5 ; les fameux successeurs de Duvergier le créateur de la machine à vapeur en 1858 , qu'ils ont amélioré avec succès ${ }^{6}$.

\section{L'application de l'électricité à l'adduction de l'eau : les usines élévatoires de Dakar}

L’introduction de l'électricité dans la colonie du Sénégal a été l'œuvre de deux ingénieurs qui ont marqué de leur empreinte son avènement dans cette colonie. Il s'agit d'Hypolite Vaubourg (ingénieur civil, domicilié à Bruyères (Voges), concessionnaire de l'électrification de St Louis en 1887) et d'André de Traz (ingénieur des Arts et Manufactures, domicilié au 19 rue Cambacérès à Paris, concessionnaire de l'électrification de Dakar en 1909).

L'électrification de Saint-Louis est intervenue en 1887 et n'a cependant pas été mise au service des usines élévatoires de Korr et de Makhana, dont les 
gros marchés d'équipement sont lancés à la même époque. En effet, la ville de Dakar a signé pour la première fois un traité de concession de l'éclairage électrique avec André de Traz en 1909, c'est-à-dire douze ans après l'éclairage de Saint-Louis.

Il est également vrai que cette première concession ne prenait pas en compte l'usage de l'énergie électrique pour les usines élévatoires de Dakar, notamment Hann et Mbao. Il a fallu un deuxième avenant, à la concession d'éclairage public de Dakar, stipulant expressément la fourniture à Hann et Mbao de l'énergie électrique nécessaire au refoulement de l'eau d'alimentation de la ville de Dakar, approuvé le 19 octobre 1909, pour que le minimum de puissance garantie pour la fourniture de courant aux usines élévatoires soit porté à $300000 \mathrm{kWh}$.

L'exploitation des usines fait l'objet d'un traité passé le 24 décembre 1915 entre la Compagnie d'Électricité du Sénégal et le Gouverneur du Sénégal. L'exploitation des usines a été bien encadrée, ce qui a rendu possible son extension.

\section{La station de Hann et l'apport de l'électricité}

La construction de l'usine élévatoire de Hann date de 1898. Celle-ci fonctionnait au début avec un moteur à pétrole. En 1904, deux machines à vapeur avec une chaudière chacune et pompes élévatoires refoulant $100 \mathrm{~m}^{3} /$ jour ont été installées pour renforcer la fourniture en eau potable de la ville de Dakar. L'usine de Hann est dotée de puits et galeries avec une mécanique composée de 12 pompes centrifuges verticales types M.60/175 à un disque. L'usine de refoulement dispose de 2 pompes centrifuges horizontales HP 150/300 M.

L'équipement électrique est composé de 12 moteurs électriques verticaux de 1,5 HP. $110 \mathrm{~V}$. $1500 \mathrm{~T}$ pour les puits et galeries; 12 transformateurs triphasés 440/110 V.2KVA; 12 disjoncteurs à maxima et minima ; 12 interrupteurs tripolaires avec flotteurs. L'usine de refoulement dispose de 2 moteurs horizontaux $30 \mathrm{HP} ; 2$ transformateurs $6600 / 440 \mathrm{~V}$ de $45 \mathrm{KVA}$.

L'établissement de lignes haute-tension pour la fourniture de la station en électricité a entraîné la réalisation d'un certain nombre de bâtiments pour son bon fonctionnement. Pour les puits et galeries, douze petits postes ont été construits. Il a suffi d'un bâtiment pour les usines de refoulement?

\section{La station de Mbao, le complément de Hann}

Le nouveau centre de captage d'eau a été créé en 1913 à Mbao. De nouveaux puits ont été créés, augmentant la quantité à $3000 \mathrm{~m} 3 /$ jour d'eau refoulée. L'équipement mécanique comprend pour les puits : 12 pompes centrifuges verticales multicellulaires types HP 70/175 à deux disques identiques à Hann. Au niveau de l'usine de refoulement, il y a 3 pompes centrifuges horizontales HP 175/300 à deux disques.

Le matériel électrique, est le même qu'à Hann au niveau des puits et galeries. Au niveau de l'usine de refoulement, il y a 2 transformateurs de $70 \mathrm{KVA}$. Le tableau de commande est équipé de 3 moteurs 30 HP.

Comme à Hann, les bâtiments pour le fonctionnement de la station de Mbao par l'alimentation électrique sont composés de douze petits postes pour puits et galeries et d'un bâtiment pour l'usine de refoulement.

Les difficultés de fonctionnement des pompes des usines élévatoires sont liées aux difficultés de recrutement du personnel, à l'impossibilité d'avoir du matériel de rechange, à l'ensablement des pompes en mois de sécheresse entraînant leur révision qui occasionne des arrêts pendant cette période. L'usure du matériel fait l'objet de réparations, par suite de l'ensablement des galeries et puits et par la teneur en sel de l'eau du centre de Hann, ayant un coût important.

Le personnel a été affecté par la grippe espagnole en 1918, 38 agents ont été touchés. L'épidémie a entraîné l'établissement d'un cordon sanitaire au km16, posant des problèmes de 
mobilité des agents d'entretien par suite de suppression des arrêts de trains à Mbao et à Hann. Cette situation a causé des difficultés dans la surveillance et le transport du matériel de rechange. Les commandes faites pour les usines élévatoires concernent des transformateurs et motopompes.

La Compagnie d'Électricité du Sénégal a cédé, pour le centre de captation d'eau de Mbao, une usine de secours constituée d'un Groupe à vapeur, de chaudières, de courroies, d'alternateur et son excitatrice et le tableau en bon état. Ceci afin d'assurer le pompage en cas d'accident grave à l'usine de Bel-Air (par exemple à la suite d'incendie ou de foudre).

L'extension de l'usine, dont la commande a été passée le 25 juillet 1914, a été arrêtée par la Première Guerre mondiale; les usines du fournisseur de la Compagnie d'Électricité du Sénégal étant à Lesquin-lès-Lille, dans la région envahie. Le matériel commandé, par l'effet de la guerre, arrive à Dakar incomplet et menace sérieusement de perturber la distribution de l'eau au niveau des usines de Hann et de Mbao. Cependant, l'usine à vapeur installée auparavant à Hann peut servir de secours en cas de nécessité à hauteur de $1800 \mathrm{~m}^{3}$.

\section{Conclusion}

La période 1880-1920 marque l'entrée des progrès techniques dans la colonie du Sénégal, depuis le début des études et réalisation du chemin de fer jusqu'à la première phase de l'électrification, en passant par les systèmes de distribution de l'eau dans les principaux centres urbains. L'ingénierie hydraulique a été le premier système technique introduit pour régler les problèmes de la vie quotidienne des populations résidant dans les centres où vivent de fortes communautés d'expatriés européens durant cette période. Les traces laissées par ces premiers équipements liés au pompage et à la distribution de l'eau constituent aujourd'hui un patrimoine technique qui témoigne de l'importance qu'occupe l'eau dans la politique de développement de la colonie du Sénégal. C'est ainsi que Saint-Louis, Rufisque et Dakar, premiers centres importants où le colon a développé son activité économique, sont plus concernés par ce programme d'ingénierie hydraulique, dont les traces sont encore visibles à travers les usines élévatoires.

Les techniques de canalisation, de construction des ouvrages (canaux, bassins, châteaux d'eau, etc.) ont été réalisées avec des matériaux importés de France souvent mélangé avec des produits locaux. Cela se fait souvent dans un style transféré à partir d'un modèle réalisé en France et adapté à la situation locale. Ces ouvrages sociaux, qui ont apporté des solutions aux problèmes liés à l'alimentation de l'eau dans les grandes agglomérations regroupant des européens et des autochtones dans la colonie du Sénégal, et qui ont servi jusqu'aux premières décennies de souveraineté de cette ancienne colonie, méritent une attention particulière. Ils constituent un patrimoine culturel et technique qui doit être sauvegardé et valorisé.

1 PAULIN, Honoré, L’Outillage économique des colonies françaises, Emile Larose, libraire-éditeur, 1913, Paris

2 Archives Nationales Section Outre-mer, TP, carton 27, dossier 4 .

3 Source gallica.bnf.fr / Bibliothèque nationale de France: exposition universelle de 1900. Les colonies françaises. Le Sénégal, organisation politique, administration, finances.....

4 FR, ANOM, SENEGAL, XII, 21 b

5 Florent Laroche, Archéologie industrielle avancée, thèse de doctorat, Ecole centrale de Nantes, 2007 http://florent.laroche.free.fr/recherche/publisSPI/IFTOMM2007_F.Laroche+BIS_V5.pdf

7 AN, Fonds anciens, AOF , Série P, P457, Bobine 145 